\title{
Supporting Material.
}
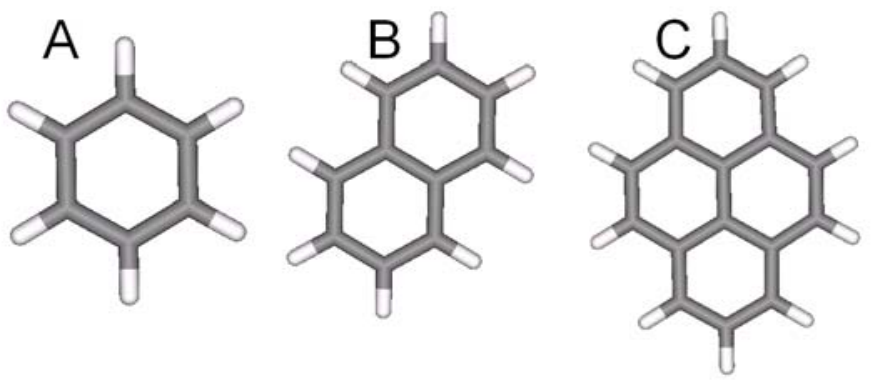

Figure S1: Structures of the studied organic linkers, A) Benzene, B) Naphthalene and C) Pyrene.

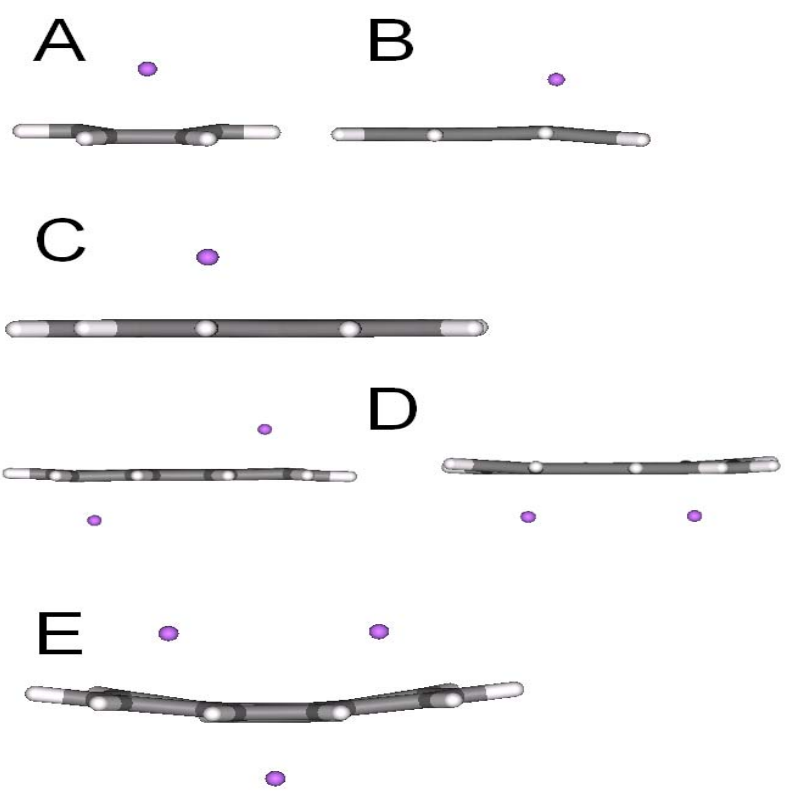

Figure S2: Structures of the Li-doped organic linkers, A) mono-Li Benzene, B) mono-Li Naphthalene, C) mono-Li Pyrene, D) double-Li Pyrene and E) triple-Li Pyrene. 